\title{
CONSTRUCCIÓN DE UN PROTOTIPO DE MONITOREO DE SEÑALES CARDÍACAS APLICANDO TECNOLOGÍA INALÁMBRICA ZIGBEE E INTERNET
}

Recibido: julio del 2018

Aceptado: marzo del 2019

Rebeca Viloria', Giovanni Bracho ${ }^{2}$, Mary Luz Torres ${ }^{3}$, Liliana Torres ${ }^{4}$

\section{Resumen}

En este trabajo se presenta el desarrollo de un sistema que permite monitorear la actividad cardíaca a personas que necesitan observación continua. Dichos registros son observables con mínimo retardo respecto al momento en que suceden, siendo almacenados y enviados por medio de una conexión a internet al implementar la tecnología inalámbrica Zigbee. Se hizo uso del software Labview para procesar los datos a partir de la señal electrocardiográfica y se desarrolló una interfaz gráfica para visualizar las señales adquiridas. Luego del proceso de adquisición, aislamiento, filtrado análogo en el dispositivo portátil, transmisión inalámbrica y recepción por parte de un $\mathrm{PC}$, se realiza el filtrado digital a la señal cardiaca adquirida para obtener la señal ECG filtrada en el PC local.

Palabras clave: internet, labview, telemedicina, zigbee.

${ }^{1}$ Ingeniero Electrónico, Universidad Popular del Cesar (UPC), Departamento de Ingeniería Electrónica.Valledupar, Colombia. viloria1996@gmail.com

2 Ingeniero Electrónico, Msc. en Ingeniería. Docente asociado, Universidad Popular del Cesar (UPC), Departamento de Ingeniería Electrónica. Valledupar, Colombia. giovannibracho@ unicesar.edu.co

${ }^{3}$ Médica y cirujana, Esp. en Pediatría. mlcardiologia@hotmail.com

4 Instrumentadora quirúrgica. Docente asociado, Universidad Popular del Cesar (UPC), Departamento de instrumentación quirúrgica. Valledupar, Colombia. Ipatriciatorres@ unicesar.edu.co 


\section{CONSTRUCTION OF A CARDIAL SIGNAL MONITORING PROTOTYPE WITH THE WIRELESS TECHNOLOGY OF ZIGBEE AND INTERNET}

Rebeca Viloria' ${ }^{1}$ Giovanni Bracho ${ }^{2}$, Mary Luz Torres ${ }^{3}$, Liliana Torres ${ }^{4}$

\section{Abstract}

This work introduces the development of a system that allows to monitor cardiac activity in people who need this continually. Those records register a minimun rate of delay in terms of the moment they happen, and they are sent through the internet by using the wireless technology Zigbee. Labview software was used to process data from the electrocardiographic signal, and we developed a graphic interface to visualize the signals. After acquisition, isolation, analog filtering of the portable device, Wireless transmission and reception in a PC, we carried out a digital filtering for the cardiac signal to obtain a filtered ECG signal in the PC. 


\section{CONSTRUÇ̃̃O DE UM PROTÓTIPO DE MONITORAÇÃO DE SINAIS CARDÍACOS COM A APLICAÇ̃̃̃O DE TECNOLOGIA SEM FIO ZIGBEE E INTERNET}

Rebeca Viloria ${ }^{1}$, Giovanni Bracho ${ }^{2}$, Mary Luz Torres ${ }^{3}$, Liliana Torres ${ }^{4}$

\section{Resumo}

Neste trabalho, apresenta-se o desenvolvimento de um sistema que permite monitorar a atividade cardíaca de pessoas que precisam de observação permanente. Esses registros são observáveis com mínimo atraso a respeito do momento em que acontecem, sendo armazenados e enviados por meio de uma conexão de internet ao implantar a tecnologia sem fio Zigbee. Utilizou-se o software Labview para processar os dados a partir do sinal eletrocardiográfico e foi desenvolvida uma interface gráfica para visualizar os sinais adquiridos. Após o processo de aquisição, isolamento, filtro analógico no dispositivo portátil, transmissão sem fio e recepção por parte de um computador, foi realizada o filtro digital ao sinal cardíaco adquirido para obter o sinal ECG filtrado no computador local. 


\section{Introducción}

En Colombia, las enfermedades cardiovasculares representan la principal causa de muerte según lo reportado por el Observatorio Nacional de Salud (ONS 2015), la población es cada vez más vulnerable a estas patologías y el monitoreo cardíaco surge como una herramienta de apoyo para la detección temprana y preventiva de estas condiciones, además de apoyo tecnológico durante el tratamiento de este tipo de padecimientos cardíacos.

Los sistemas de monitoreo de señales electrocardiográficas (Davis, 2008) en pacientes tienen actualmente un área de cobertura limitada al centro hospitalario, los desarrollos efectuados con sistemas de monitorización incluyen electrocardiógrafos que se conectan por medio físico a un computador (Vidal \& Gatica, 2010), electrocardiógrafos inalámbricos (Quintero, et al., 2018), sistemas de monitoreo que integran módulos GPS, GSM/GPRS y Zigbee (Valenzuela, et al., 2015), entre otros.

La expansión de las tecnologías de información genera impactos que no son ajenos a la medicina y a la salud (Murga, 2015), es aquí donde la telemedicina se vuelve significativa al establecer una conexión entre el personal médico-asistencial en un centro hospitalario y los pacientes en sus hogares, gracias al uso de sistemas de telecomunicaciones $(\mathrm{Mu}-$ rillo, et al., 2016). Este tipo de sistema tecnológico integrado para la prestación de servicios de salud permite la detección, el monitoreo y el seguimiento de sucesos o eventos en la condición mé- dica del paciente de forma remota, al conservar un nivel de equivalencia en sus características comparable al proceso de forma presencial, donde el médico especialista obtiene la mayor información posible del paciente basándose en los resultados arrojados por los equipos biomédicos (Herrera y Periche, 2017).

Una de las herramientas básicas en el diagnóstico de trastornos y enfermedades del corazón es el electrocardiograma, el cual es realizado comúnmente por su simplicidad, bajo costo y por tratarse de una técnica no invasiva (Carrión, et al., 2009). Este procedimiento consiste en el registro gráfico de la actividad eléctrica que se genera en el corazón. Su análisis proporciona importante información que complementa el conjunto de datos que se registran en la historia clínica de un paciente, con un objetivo diagnóstico y después de una exploración física detallada (Portillo, 2011).

Los equipos de monitoreo cardíaco disponibles en la actualidad realizan el proceso de registro de información de forma limitada, debido a que los datos son almacenados automáticamente por eventos determinados por cierto periodo de tiempo. El médico especialista en cardiología se encarga de analizar los datos una vez finaliza el periodo de monitoreo, pero no en tiempo real, impidiendo una reacción rápida ante una emergencia causada por una anomalía cardiaca.

\section{Metodología y desarrollo}

En este trabajo se ha desarrollado una metodología de forma ordenada y se- 
cuencial, plasmada de la siguiente manera:

- Fase de investigación y documentación

- $\quad$ Fase de diseño
- Fase de implementación

- Evaluación de resultados

Definiendo así un diseño metodológico representado en la figura 1, para cumplir con los requerimientos técnicos y obtener el resultado esperado.

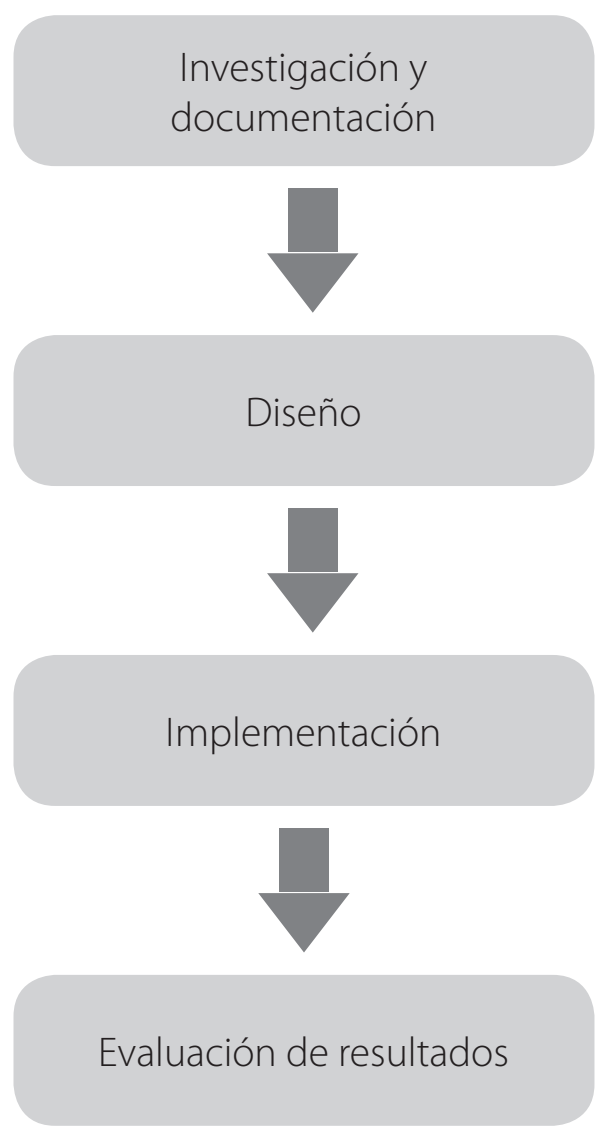

Figura 1. Modelo de trabajo Fuente: elaboración propia.

\section{Fase de investigación y documentación}

Se realizó una investigación de los elementos, los factores y las variables reque- ridos para la ejecución de este trabajo, entre los cuales destacan: fundamentación teórica del monitoreo cardíaco ambulatorio, bases teóricas del sistema eléctrico del corazón y conocimientos 


\section{Fase de diseño}

Para esta fase se elaboró inicialmente un diagrama de bloques donde se proyectó el comportamiento del dispositivo ya terminado, en la figura 2 se observa el diagrama de bloques de funcionamiento del prototipo. Se concibió un prototipo de monitoreo cardíaco ambulatorio utilizando componentes de bajo consumo de potencia; el dispositivo diseñado captura las señales eléctricas del corazón y las envía a un servidor remoto por medio de la tecnología inalámbrica Zigbee, lo que garantiza un monitoreo constante que hace uso de internet, la información capturada es enviada de forma continua o en periodos de tiempos configurables definidos según el criterio del médico especialista.

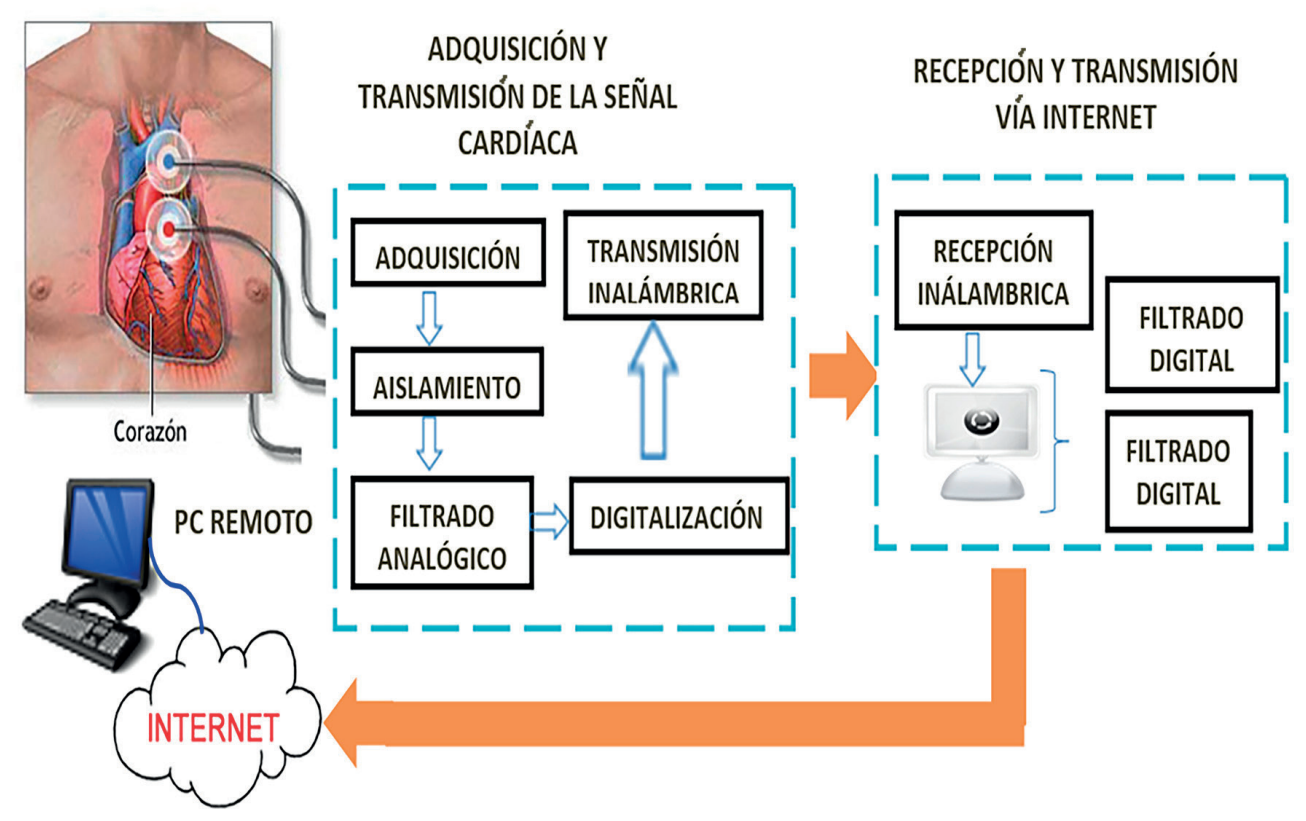

Figura 2. Diagrama de bloques de funcionamiento del prototipo Fuente: elaboración propia.

\section{Fase de implementación}

Para la adquisición, el procesamiento y la visualización de la señal ECG se utilizó el software Labview, ya que ofrece bibliotecas integradas para análisis avanzado y visualización de datos y facilita la creación de instrumentación virtual, con el que se aprovechan funciones análisis de frecuencia, ajuste de curvas,

filtrado, entre otras; además de realizar análisis en línea en tiempo real en señales adquiridas. El dispositivo diseñado plasma como lineamientos la miniaturización en conjunto con la portabilidad, ya que la señal ECG no es almacenada, sino que es transmitida después que ha sido filtrada y digitalizada. En la figura 3 se puede apreciar un conjunto de los componentes más relevantes utilizados en la construcción del prototipo. 


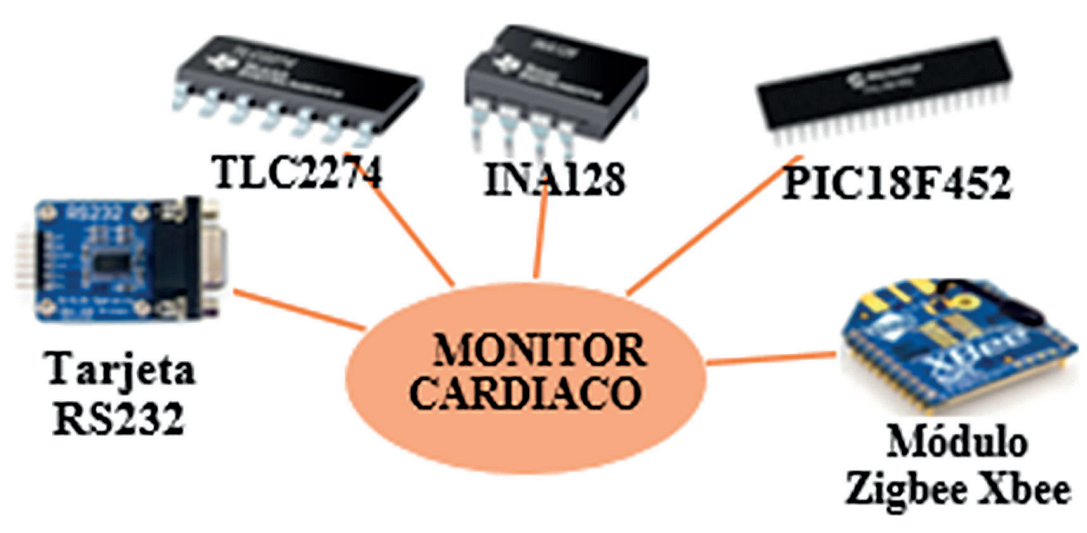

Figura 3. Materiales más relevantes utilizados en el desarrollo del monitor cardíaco Fuente: elaboración propia.

En la fase de implementación se siguieron las siguientes etapas para el desarrollo del prototipo:

\section{Adquisición aislamiento y tratamiento de la señal electrocardiográfica}

La señal cardiaca es recolectada a través de los electrodos adheridos al cuerpo del paciente, esta debe ser amplificada debido a su bajo nivel de voltaje por un amplificador de instrumentación, luego debe ser aislada eléctricamente para protección del paciente con un opto-acoplador y posteriormente realizarse un proceso de cancelación de ruidos y señales eléctricas no deseadas por medio de un filtrado pasa-banda, comprendido entre $0,05 \mathrm{~Hz}$ y $100 \mathrm{~Hz}$, por ser este el rango en que se definen las señales EKG.

\section{Amplificador de instrumentación}

Para obtener la señal del EKG y realizar un prototipo de bajo costo se utilizaron electrodos de superficie, ya que son más económicos que los de inserción y brindan comodidad al paciente (Electromedicina, 2011). Se deben ubicar unos electrodos en puntos estratégicos del cuerpo según la derivación electrocardiográfica que se seleccione, los cuales captarán impulsos eléctricos que deben ser amplificados por su baja intensidad. Para este fin se seleccionó el amplificador de instrumentación INA128, por su alto rechazo al modo común (CMR - 120dB), de propósito general y bajo consumo de energía que ofrece excelentes características.

En la figura 4 se puede observar el esquema de adquisición de la señal EKG con el integrado INA128P. La ganancia con la que se ha configurado el amplificador de instrumentación para amplificar la señal proveniente del corazón a través de los electrodos es de $1.000 \mathrm{~V} / \mathrm{V}$. 


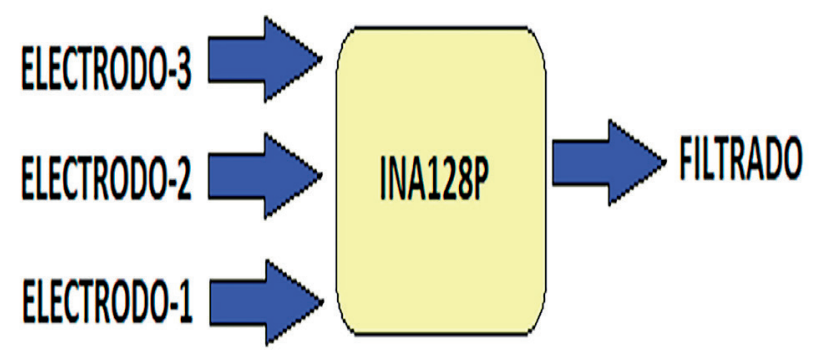

Figura 4. Esquema adquisición señal EKG con el integrado INA128P

Fuente: elaboración propia.

\section{Filtrado}

Después de adquirir y amplificar la señal en la etapa anterior, es necesario minimizar el ruido y las señales eléctricas indeseadas. Al tener en cuenta que solo son requeridas las componentes de señal en un rango de frecuencias comprendido entre $0,05 \mathrm{~Hz}$ y hasta $100 \mathrm{~Hz}$, se implementó un filtrado pasa-banda constituido por un filtro pasa-altos y un filtro pasa-bajos, diseñados para el rango de frecuencias anteriormente mencionado. El amplificador opera- cional seleccionado para el filtrado es el cuádruple OPAM TLC2274 de Texas Instruments, cuya principal característica es ser un amplificador de bajo ruido y rail to rail, es decir que el voltaje de salida del operacional nunca alcanzara a VDD (voltaje de alimentación positiva), sino que será ligeramente inferior. Adicionalmente, proporciona un ancho de banda de $2 \mathrm{MHz}$ y una corriente de polarización de entrada típica de $1 \mathrm{pA}$. En la figura 5 se aprecia un diagrama de bloques del filtro pasa-banda.

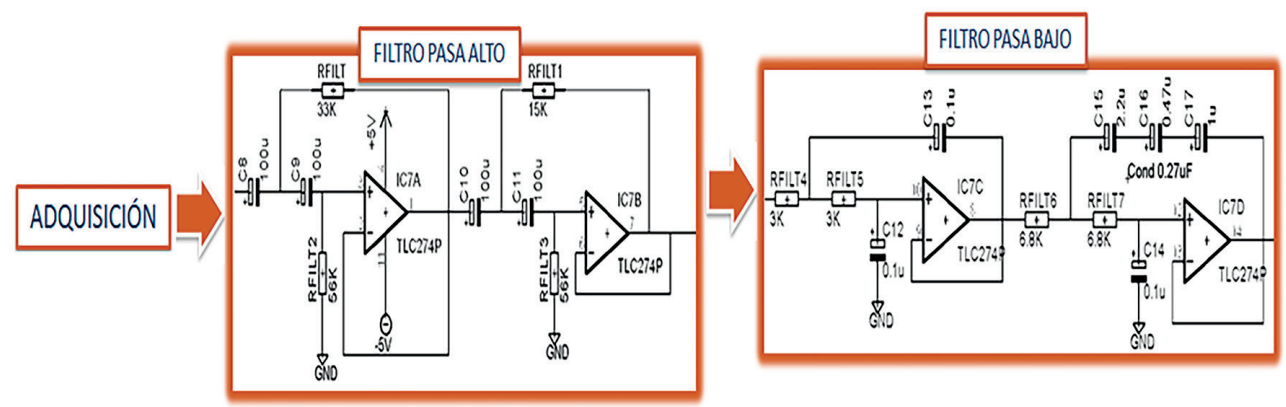

Figura 5. Esquema de filtro pasa-banda con el OPAM TLC2274

Fuente: elaboración propia.

\section{Acondicionamiento}

Luego de ser filtrada, la señal electrocar202 diográfica debe ser digitalizada para ser transmitida posteriormente de forma inalámbrica. Previo a la transmisión, para que el microcontrolador pueda realizar adecuadamente el muestreo de la señal EKG, esta debe acondicionarse a niveles de voltaje comprendidos entre 0 y $5 \mathrm{~V}$. 
El OPAM LM358 de la empresa Texas Instruments fue seleccionado para acondicionar la señal proveniente del filtrado pasa-banda y, por tanto, garantizar que los niveles de voltaje no superen los $5 \mathrm{~V}$ ni que bajaran de los $0 \mathrm{~V}$. En la figura 6 se observa el esquema acondicionamiento de la señal EKG con el LM358.

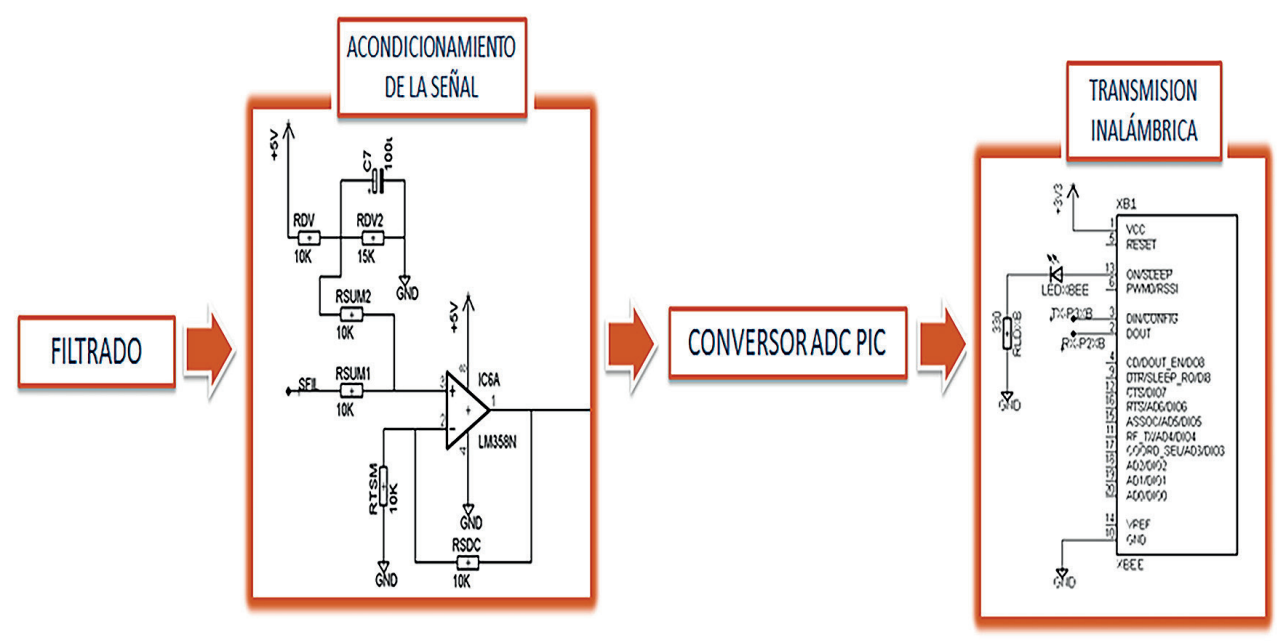

Figura 6. Esquema de acondicionamiento de la señal EKG con el LM358

Fuente: elaboración propia.

\section{Selección del microcontrolador}

La selección del PIC 18F452 se debe a su conversor de datos de 10 bits, este PIC cuenta con una memoria de $32 \mathrm{k}$ que permite adquirir y transmitir los datos al módulo Zigbee y monitorear otros procesos del prototipo, con el fin de garantizar la continuidad de la transmisión de la señal.

\section{Transmisión y recepción inalámbrica}

Posterior al muestreo realizado por parte del conversor ADC de 10 bits del PIC18F452, la señal de EKG es transmitida inalámbricamente por medio de un módulo Zigbee configurado como END DEVICE AT y recibida por un módulo Zigbee configurado como COORDINADOR AT, el cual está conectado por medio de una tarjeta RS232-USB a un computador.

\section{Fuente de voltaje}

Para la alimentación del dispositivo transmisor del prototipo de monitoreo cardíaco se seleccionó una batería recargable de Ion de Litio de 9V / 350 mAh, ideal para un sistema portátil de bajo consumo.

La fuente primaria de voltaje es la batería de $9 \mathrm{~V}$, la cual por medio del circuito integrado ICL7660 es convertida a un valor negativo, el regulador LM7905 se encarga de estabilizar y permite entregar de forma constante $-5 \mathrm{~V}$, los cuales son requeridos por los circuitos integrados de alimentación dual junto a los $5 \mathrm{~V}$ regulados por un LM78LS05, tra- 
duciéndose este sistema en una fuente dual óptima.

\section{Elaboración del software aplicativo}

El funcionamiento del software comienza con el proceso de adquisición de datos en el VI (instrumento virtual) o VISA (del inglés Virtual Instrument Software Architecture) serial de Labview, el cual permite configurar los parámetros como baudios de transmisión, bits de datos, paridad y control de flujo que harán que el proceso de adquisición de datos sea correcto. Luego de realizada la adquisición de datos se hace la representación gráfica de las señales cardíacas para someterlas a un proceso de filtrado que favorecerá la visualización óptima de la señal.

\section{Evaluación de resultados}

Para finalizar con la fase de desarrollo del prototipo, se creó el manual de instrucciones para el correcto manejo del hardware y del software que hacen parte del proyecto y se realizaron las pruebas necesarias para determinar la confiabilidad del sistema.

\section{Resultados}

\section{Visualización de la señal}

La señal adquirida es procesada digitalmente, para visualizarla se utiliza la interfaz de adquisición de datos, a su vez, esta señal está siendo almacenada y transmitida a través de internet mediante la aplicación "Cliente-Servidor", la cual debe ser configurada para que exista comunicación y el proceso de transmisión sea satisfactorio. En las figuras 7 y 8 se puede apreciar la interfaz de la aplicación del servidor y la interfaz de la aplicación del cliente respectivamente. La figura 9 muestra la señal cardiaca en el computador local, la cual ha pasado por el proceso de adqui $\neg$ sición, aislamiento y filtrado análogo, transmisión inalámbrica y filtrado digital. Para la correcta configuración de la aplicación se creó el manual de instrucciones para el manejo del software, de igual manera se diseñó un manual para el adecuado uso del hardware que hace parte del proyecto, se realizaron las pruebas necesarias en compañía del médico especialista para determinar la confiabilidad del sistema. 


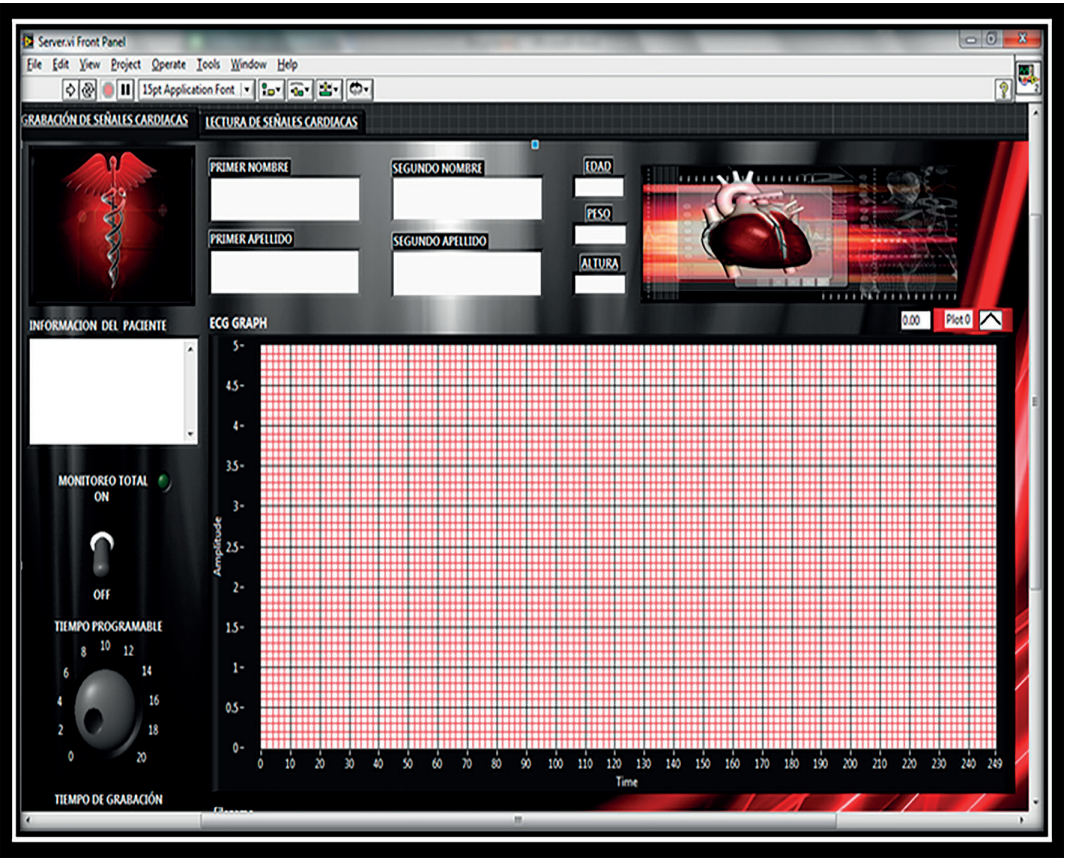

Figura 7. Interfaz de la aplicación del servidor Fuente: elaboración propia.

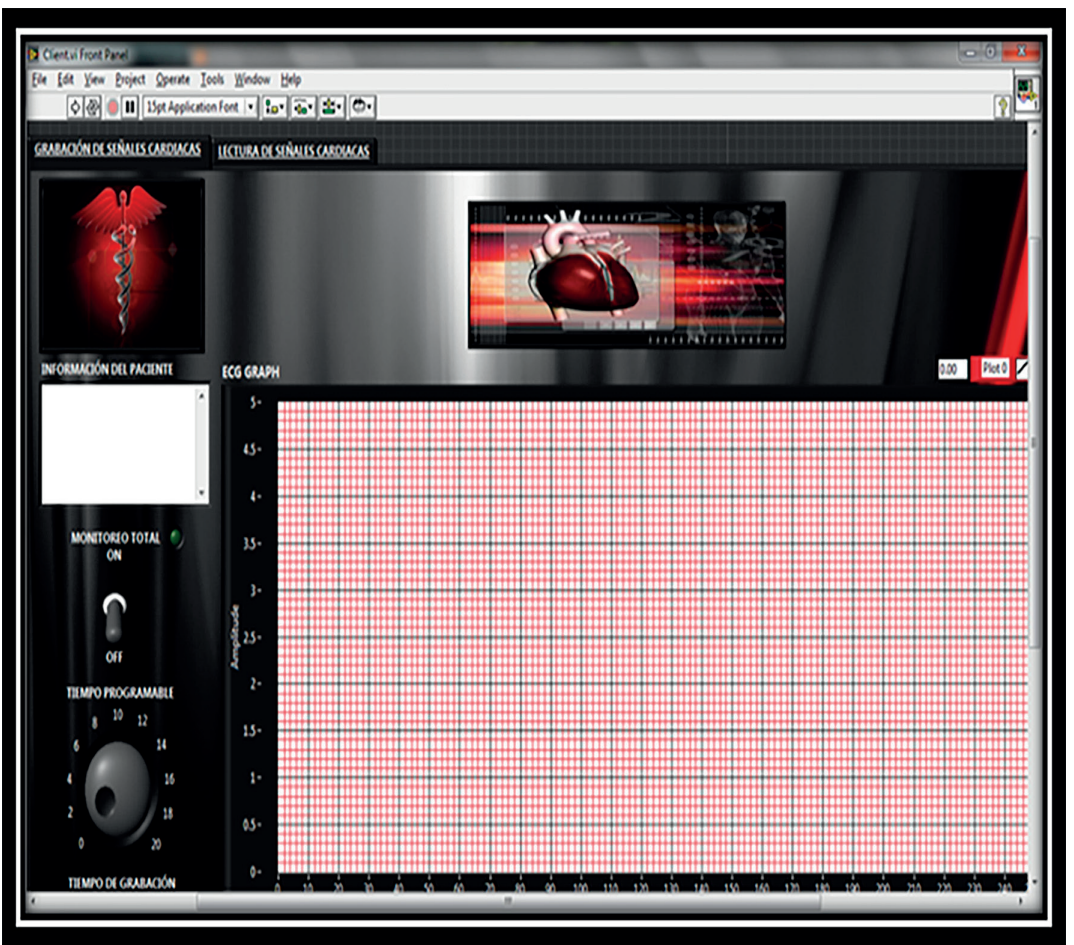

Figura 8. Interfaz de la aplicación del cliente 


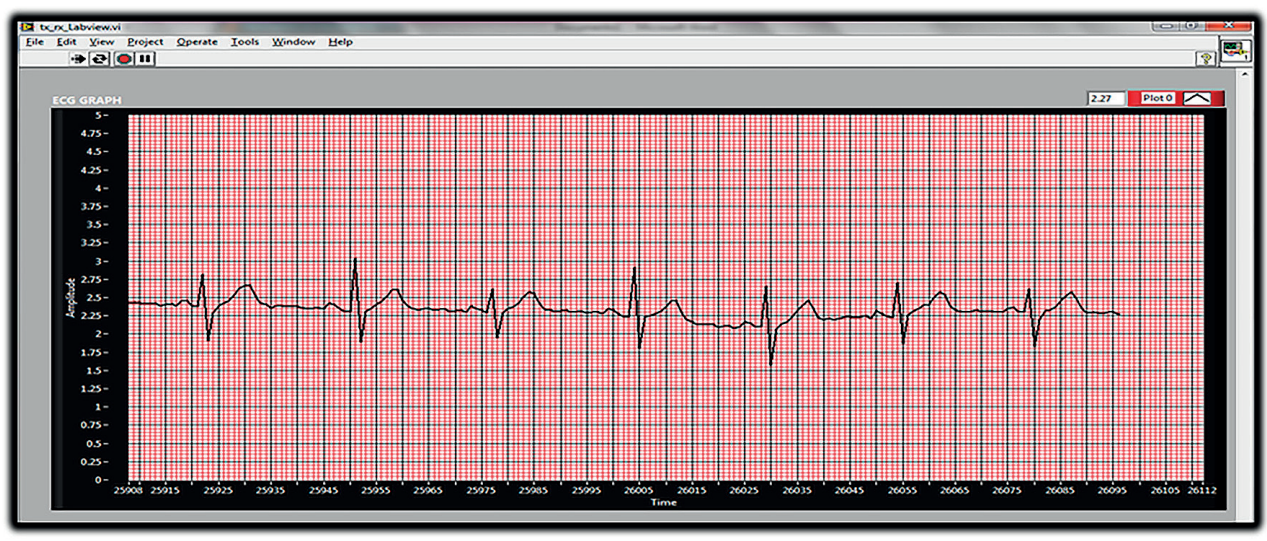

Figura 9. Primeras pruebas de la señal cardíaca adquirida en el PC con el software Labview Fuente: elaboración propia.

\section{Evolución del proyecto}

Este proyecto da origen a una segunda fase, la cual se encuentra actualmente en periodo de diseño y desarrollo, donde se pretende actualizar y ampliar características del mismo, por ejemplo, aplicar la tecnología Bluetooth BLE que posee características de última generación como tamaño reducido, bajo costo, ahorro de energía y compatibilidad con la mayoría de dispositivos inteligentes (Collotta y Pau, 2015). Otra evolución que se pretende lograr sería el desarrollo de una aplicación para dispositivos móviles Android que reemplazará la aplicación del cliente creada en Labview. Con la evolución propuesta se pretenden obtener mejoras como un menor consumo de energía, un tamaño reducido, una mayor portabilidad y una mejor ergonomía, etc.

\section{Conclusiones}

Este trabajo de investigación y de desa206 rrollo propone una forma de monitoreo cardíaco ambulatorio que implementa la tecnología inalámbrica Zigbee e internet, al enviar la información recolectada de forma continua o por periodos de tiempos configurables hacia centros médicos, de acuerdo con los criterios de los médicos especialistas en esta área.

La utilización del prototipo no compromete el estado clínico, la salud ni la seguridad de los pacientes o de quienes estén en contacto con los mismos, por lo tanto, garantiza la seguridad del paciente, ya que cuenta con los parámetros de protección requeridos tales como circuitos de protección y de aislamiento que lo califican como un dispositivo confiable.

Cuenta con un sistema de transmisión inalámbrico que permite el desplazamiento del paciente dentro de un perímetro específico sin incomodar a la persona portadora de este equipo de monitoreo, con las mejoras propuestas, como la incorporación de Bluetooth BLE, se permitirá un rango más amplio de desplazamiento, siempre y cuando se mantenga el dispositivo móvil dentro de un perímetro establecido. 


\section{Referencias bibliográficas}

Carrión, P., Ródenas, J., Rieta, J. y Meléndez, C. (2009). Telemedicina.: Ingeniería biomédica. Cuenca: Universidad de Castilla La Mancha.

Collotta, M. \& Pau, G. (2015). A solution based on bluetooth low energy for smart home energy management. Energies, 8(10), pp. 11916-11938.

Davis, D. (2008). Interpretación del ECG: $\mathrm{Su}$ dominio rápido y exacto. Buenos Aires: Editorial Medica Panamericana.

Electromedicina, A. (mayo 2011). Bioingeniería y Biosensores. Recuperado de http://aplicacioneselectromedicina.wordpress.com/

Herrera, F. \& Periche, F. (2017). Sistema de Telemedicina UdC: Un nuevo paradigma en la atención médica colombiana para el sur de Bolívar. Informática y Sistemas: Revista de Tecnologías de la Informática y las Comunicaciones, 1(1), pp. 1-2.

Murga, L. (2015). Estudio del sistema mysoul y de la telemedicina orientada a la atención de las afectaciones cardíacas, para el diseño de la aplicación móvil de visualización y análisis de las señales ECG para médicos (Doctoral dissertation). Universidad de Guayaquil, Ecuador.
Murillo, M., Cardoza, L., López, R. y Cruz, C. (2016). Cifrado caótico simétrico de ECG y EEG para aplicaciones en telemedicina. XVII CLCA Latin American Conference of Automatic Control, 612(14), , pp. 612-617.

Portillo, M. (2011). Electrocardiografía: Técnica de interpretación básica. VI foro de pediatría de atención primaria de Extremadura. Recuperado de http://www. spapex.es/pdf/taller_ekg.pdf

Quintero, J., Tabares, S. y Perdomo, J. (2018, noviembre). Prototipo Electrocardiógrafo Inalámbrico para la detección de enfermedades que desencadenen la muerte súbita, con software de diagnóstico médico aproximado. Memorias de Congresos UTP, 1(1), pp. 18-23.

Valenzuela, O., López, J. y García, E. (2015). Sistema de monitoreo para pacientes de alto riesgo integrando móduIos GPS, GSM/GPRS y Zigbee. Tecnura, 19, pp. 98-111.

Vidal, C. y Gatica, V. (2010). Diseño e implementación de un sistema electrocardiográfico digital. Revista Facultad de Ingeniería, Universidad de Antioquia, 55, 99-107. 